\title{
Disturbances of neurotransmitter metabolism in anorexia nervosa
}

\section{By A. H. CRISP, Academic Department of Psychiatry, St George's Hospital Medical School, Tooting, London SWI 7}

My understanding of anorexia nervosa is that it is ultimately a biological solution to an existential problem for an adolescent female and her family in the classical case. It is not seen by the individual concerned as an affliction but rather, perhaps, as adaptive and egosyntonic. In order to protect her status the so-called patient will often successfully conceal major aspects of her feelings and behaviour. She shares this propensity with many other similarly-motivated people, for instance many people with neurotic illness, and the alcoholic.

One popular construction put upon her syndrome is that she has lost her appetite and that this is the explanation for her reduced food intake. Another view, perhaps bearing in mind such features as her excessive preoccupation with food and involvement in its preparation, is that she is liable to an excessive and premature satiety. In my view neither of these formulations does sufficient justice to the complexity of the experiential aspects including the determinants of this condition. Correspondingly, there are as yet few signs that effective pharmacological manipulation of anorexia nervosa is a near reality.

In recent years two principal theoretical contributions have been made in terms of possible disturbances of neurotransmitter metabolism in the condition. Mawson (1974), in a closely-argued thesis, proposed that the syndrome of loss of appetite or excessive satiety or both could be explained on the basis of a subcortical, probably hypothalamic, defect involving progressive depletion of brain noradrenalin or dopamine or both. He suggested that treatment with l-DOPA might be effective. Mawson's (1974) grasp of the clinical nature of the condition extended to an understanding that patients often seem purposefully to resist eating. He quotes Russell (1970) as saying that this does not rule out a primary hypothalmic lesion. More recently Redmond, Huang, Baulu, Snyder \& Maas (1977) have proposed, again on the basis of animal studies, that the apparently-increased satiety in anorexia nervosa might be due to hyperactivity of postsynaptic noradrenergic receptors. Commenting on Mawson's (1974) dopamine theory they conclude that the use of 1DOPA is doomed to failure because of the likelihood that it would also increase noradrenalin activity. They suggest that the most effective pharmacological approach would be the exhibition of $\alpha$-adrenergic blocking agents.

At this stage I will make some further comments as a clinician.

(I) It is in my view likely to continue to be difficult to set up animal models which reflect the complex factors governing behaviour in anorexia nervosa. 
(2) Many anorectics in fact experience an excessive desire for food and for its ingestion. Personally they often seek appetite supressants. Clinically approximately one-third of the population of hospital-contact anorectics are sporadically overeating. Satiety is something they desperately seek, but often without avail, through the ingestion of huge quantities of non-energetic or low-energy substances. If instead they find that they have ingested large quantities of energy then they vomit them or purge excessively or both. These patterns give one a clue to their more fundamental experiential need, which is to maintain a low bodyweight. Indeed anorexia nervosa is not in my view primarily an eating disorder except in a specific other sense that I will come to later.

(3) Next, and relatedly, assuming that eventually it will become possible to manipulate pharmacologically the physical aspects of the condition by drugs affecting: (a) ingestive behaviour in such a way as to unbridle it biochemically; whilst (b) at the same time overcoming the individual's resistance to eating; (c) suppressing vomiting and also the ingestion of purgative drugs (a fairly tall order), then this in my view will often have immediately damaging psychological effects consequent upon the helpless experience of unavoidable weight gain. It is also likely in the long run to promote an even more severe state of anorexia nervosa in the individual concerned, provided of course she can in time come to avoid consuming the previously-mentioned drug by some deception or other (e.g. by her coming to maintain either an even greater extent of abstinence or, more sinisterly, by her persisting instead with a 'bingeing' eating style, but accompanied now by vomiting and purging in order to maintain low weight control). At the moment, the substances that come closest to promoting increased appetite and especially carbohydrate craving, which is the concern of the anorectic, are amitriptyline (Paykel, Mueller \& de la Vergne, 1973) and insulin.

In the rest of the paper I will first attempt to sustain such provocative assertions (by making further reference to my clinical views and our related research) and then also indicate ways in which I think anorexia nervosa may come eventually to provide us with a unique model for the study of cerebral neurotransmitters and also the more general regulation of food intake.

( 1 ) Anorexia nervosa, in my view, is rooted in certain constitutional and growth factors interacting with psychosocial conflicts of adolescence and their identification in terms of postpubertal fatness. Psychologically speaking the primary pathology has to do with fatness and not with food as such (Crisp, 1973, 1977).

(2) It reflects a psychologically-necessary stifling of biological maturation, in fact a regression, through a reversal of the pubertal biological process, back to what is in many ways a childlike biological state. The state is achieved through the facilitation of and, in the first instance anyway, maintenance of dietary carbohydrate avoidance, this being the mechanism that generates the regression together with its psychologically-relieving effects for those in conflict and without other resource (Crisp, 1967a,b, 1970). The initial carbohydrate avoidance is part of a much more widespread female adolescent behaviour pattern. 
(3) Thus the majority of adolescent females in Europe and North America are attempting to curb their fatness by dieting. Whilst obesity is over-represented in these populations it is not a sufficient explanation for this behaviour. The fatness in question, conferred on the female at puberty, has sexual significance. Fashion is not a sufficient explanation for the current craze with dieting (fashion merely reflects the underlying needs and wishes of society). If such dieting is more common than it used to be this probably has to do with (a) the higher and probably now optimal, though still individually variable, growth rate of recent generations; (b) the absence of cultural and institutional transitional control systems with respect to adolescent sexual behaviour in our present-day society and at a time of accelerating cultural change (this tending to generate greater stress in middle-class families than other family groups); (c) an individually-high growth rate with an early major pubertal growth spurt, early menarche, voracious appetite and plumpness which characterize though are not necessary features of the build-up to anorexia nervosa. When present they serve to remind us of the importance of the appetitive antecedents of the condition. Unquenchable appetite may become especially associated with biological sexual differentiation, thrust and ripeness at a time when postpubertal psychological differentiation is often embryonic (the adult search for identity may have barely begun.) In the sense that life-long or good overnutrition will have promoted such rapid growth, food and its personal childhood and family psychological relevance can be seen as the primary agent, but it is the intervention of puberty which has precipitated the concern about fatness and shape and hence body-weight. The big appetite characteristic of this period, usually with carbohydrate craving as a prominent feature, appears to be similar to the experience many people have when consuming amitriptyline or when given small doses of insulin. Both these 'treatments' are contra-indicated in anorexia nervosa in my view. In particular, the use of 'modified insulin treatment' is contraindicated. Abstaining anorectics, at least, already have high levels of resting insulin and hypoglycaemia. The 'anorectic-to-be,' and for that matter the established anorectic, might be much better served by a drug having the opposite effect, providing them thereby with a sense of better ingestive control.

(4) The hypothalamus and the more extensive subcortical systems, of course, play a major role in mechanisms of anorexia nervosa. However, I remain to be convinced that, apart from the contribution that stems from such constitutional factors as I have mentioned previously, they play any major primary part in the genesis of anorexia nervosa. I have always emphasized what I regard as being unique about the starvation that is characteristic of the nuclear (abstaining) form of anorexia nervosa, i.e., that it is of carbohydrate. From a clinical standpoint it has long seemed to me that this not only contributes to the widespread metabolic qualities that are a hallmark of the condition but that it could well also account for the early onset of amenorrhoea. I am impressed by the role of dietary factors, operating through the medium of reducing basal metabolic rate (BMR), in generating the amenorrhoea in anorexia nervosa. In a population of our patients premorbid weight was $6 \mathrm{I} \pm \mathrm{II} \mathrm{kg}$ (matched population mean $\pm S E$ weight 
$55 \pm 3.7 \mathrm{~kg}$ ). Weight at time of last menstrual period was $52 \pm 6 \mathrm{~kg}$ and weight at resumption of menstrual bleeding was $53 \pm 6 \mathrm{~kg}$ in that group which recovered (Crisp \& Stonehill, I 971). I understand that it has now been shown that restriction of carbohydrate in the diet produces a greater drop in BMR than does dietary restriction of isoenergetic amounts of fat and protein.

We have also demonstrated, in abstaining anorectics, that there is a fairly precise weight threshold of approximately $44 \mathrm{~kg}$ below which the pituitary fails to respond to stimulation with LH-RH. In anorectics who 'binge' and vomit and whose basal metabolic status is correspondingly increased compared with abstaining anorectics for any given body-weight, then those thresholds are lower. Menstrual bleeding, which in a premorbid obese girl may have occurred at a bodyweight of for example $5^{8} \mathrm{~kg}$, will return under these conditions as weight is gained at approximately $48 \mathrm{~kg}$. Moreover, such individuals can sometimes fall pregnant at low body-weights and without having resumed menstruation; indeed the dissonant metabolic status of this group of patients who may eat incessantly and yet maintain low weight by vomiting and purging seems to me almost to defy nature. Thus the abstaining anorectic is highly aroused and with a very low BMR (qualities seemingly consonant with such a severe extent of starvation). However, the anorectic who maintains the same low weight by 'bingeing' and vomiting is often seemingly even more highly aroused and unsatiated but at the same time she has a higher BMr. Experientially she has a voracious appetite and a permanent and shameful sense of imminent loss of control rooted in her conviction that she could rapidly become obese. Certainly this low-weight-control style provides a very different basic metabolic picture, such that all biological studies need to take it into account and to allocate accurately subjects being studied to one or other group of ingestive food disorders if they are to make sense of their findings (Crisp, 1967a, I977a).

(5) I will not dwell here on the psychological conflicts that I believe precipitate the condition, nor on the family and personal psychological antecedents to the condition. I have elaborated extensively on these aspects elsewhere (see Crisp, $1977 b$ ). What I wish to do next is to comment on certain of the psychological behavioural and metabolic features within the condition (they are inextricably interwoven). (a) Anorectics display the behavioural and psychological features of hungry and starving people. They are alert, restless by day and night, they hoard (and hence sometimes steal) things including food and they are totally preoccupied with thoughts of food. Whilst such orientation has obvious biological survival value, it is the ultimate irony for the anorectic, striving to avoid and forget about food in the presence of plenty. It leads to much of her defensive behaviour, such as social withdrawal, ritualistic behaviour in relation to eating, and excessive involvement in school work. (b) The anorectic will often deny to others that she is hungry or that she is concerned about her weight. As time passes, her appetitive experience has become distorted in association with the progressive shrinking and atrophy of the stomach and small bowel or its intermittent or sustained exposure to large quantities of food which is then vomited or both. Acute gastric dilatation 
can occur. Gastric and small bowel motility usually persist in a normal fashion. Probably partly as a result of her small stomach, and associated significantly small parietal cell mass, the abstaining anorectic comes to feel bloated after eating only a small quantity of food (Crisp, 1970). She may then fear that she looks pregnant. However, the importance of the psychological and social settings for this experience of being bloated is nowhere more clearly demonstrated than in the treatment situation. At any one time we have seven or eight patients with anorexia nervosa in in-patient treatment. These individuals have made a psychological commitment to change. Such an anorectic, who may have been more or less abstaining from eating anything but small quantities of non-carbohydrate food for anything from 3 to 20 years, can settle down and immediately consume $12.6 \mathrm{MJ}$ (3000 kcal)/d including normal amounts of carbohydrate in the form of normal hospital meals. (c) Perceptually, anorectics are characterized by reporting gross self-over-estimates of their body widths. Russell (1970) first showed this in his work with Slade (Slade \& Russell, 1973). It is now clear that anorectics share this propensity not only with obese females but also with many normal adolescent females, although few of the latter display it to the same extent as the anorectics. I am inclined to the view that this quality relates to the adolescent female's concern with her shape, with her fear of loss of control over her shape, her sexual and her interpersonal destiny, and that this is a particularly prominent diathesis in those who are obese and those who go on to develop anorexia nervosa. It may have its roots in fat cell processes (Crisp \& Kalucy, 1974).

We have demonstrated that in anorectics this over-estimate can be modified by suggestion; that it is sensitive to the perception of carbohydrate, and to a nonenergetic sweet taste which is assumed by the anorectic to be energy-containing. These latter two stimuli, which generate reported increases in self-estimates of body widths in anorectics, significantly characterize them when they are compared with normal female subjects matched for age. Finally, anorectics have a normal capacity to detect sweetness by taste (Lacey, Stanley, Crutchfield \& Crisp, 1977). (d) It has been suggested from animal experimental work that fasting reduces sympathetic activity (Young \& Landsberg, 1977). Fasting certainly reduces BMR in humans. There is no thermic effect of food; lean body mass is eventually reduced and, as I have argued elsewhere, there is, in the face of carbohydrate restriction in particular, an early biological retrenchment to a state wherein reproductive capacity is 'switched off.' However, we are all equally aware that not eating arouses us whilst a good meal effectively puts us to sleep despite the most stimulating of lectures. It has been my contention that the restlessness and other behavioural hallmarks of anorexia nervosa reflect this effect of starvation. I reported Io years ago (Crisp, I $967 a$ ) that early-morning waking, as part of a major disruption of sleep in the second half of the night, is a feature of anorexia nervosa. Early-morning waking has for long been regarded by some as pathognomonic of a particularly severe kind of depressive illness, though in fact not all such people experience it. However, when they do they complain bitterly of it (they would rather be oblivious especially in the early hours of the morning). Early-morning 
waking is a much more universal feature of anorexia nervosa, occurring as it does in young adults who would normally be expected to sleep a solid $8 \mathrm{~h}$ or so. However, anorectics do not complain of it, they will not usually volunteer its existence and they may even conceal the fact. The anorectic likes to be alert and active and may use the early hours for secret activities. Our early studies led us to the conclusion that such sleeplessness was unrelated to mood but was related to the nutritional state. In pursuit of this we studied not only anorectics gaining weight but also obese subjects losing weight; also while mood was monitored, we studied psychiatric patients gaining and losing weight within the illness irrespective of diagnostic label (Crisp \& Stonehill, 1973, 1976).

It seemed to me that $4 \mathrm{~h}$ solid sleep was all that the anorectic was allowed. It was tempting to see this as biologically regressive too. Since the anorectic did not then assuage her hunger or improve her nutritional state she thereafter remained wakeful and active until, exhausted at the end of the day, she once more reverted to sleeping for a few hours.

Over the last to years we have pursued research into nutrition and sleep more widely on the basis that this might eventually allow us to penetrate to some of the central mechanisms governing energy balance. The collaborators with me in this work have been Drs Stonehill, Kalucy, Chen, Lacey, Gaitonde, Miss Margot Hartmann and a number of research students attached from the University of Surrey.

In the first instance we studied a further anorectic population in respect of its nocturnal motility (Crisp, Stonehill \& Fenton, 1970). We found such motility to be significantly reduced after restoration of 'matched-population' mean weight. Next we examined a few obese subjects in more detail during their major weight reduction (Crisp \& Stonehill, 1970). We confirmed that nocturnal motility and daytime activity increased and total sleep time decreased as weight was lost. The major change in these respects coincided with loss of menstruation. One of these patients was studied longitudinally with sleep electroencephalograms (EEG) (Crisp, Stonehill, Fenton \& Fenwick, 1973). Major weight loss was associated with reduced 'slow-wave' sleep in contrast to the findings of MacFadyen, Oswald \& Lewis (1973) in respect of less severe and prolonged starvation (a mean weight loss of $5 \mathrm{~kg}$ ), but in accord with Anokhin's (1961) finding that in hungry cats, an EEG arousal pattern is replaced by a record of pronounced synchrony similar to that of 'slow-wave' sleep when the stomachs of the animals are filled with milk and glucose administered intravenously. This finding was illuminated further when we studied, by means of EEG-sleep recordings, ten anorectics as they were renourished, first at low body-weight (mean $37.5 \mathrm{~kg}$ ), then at $15 \%$ below 'matchedpopulation' mean weight (mean $44.2 \mathrm{~kg}$ ), and finally at 'matched-population mean' weight (mean $52.0 \mathrm{~kg}$ ). At low body-weights subjects displayed limited and restless sleep in association with significantly low plasma prolactin levels (Kalucy, Crisp, Chard, McNeilly, Chen, Carruthers \& Zilkha, 1977), especially during the last $4 \mathrm{~h}$ of the recording. Following partial restoration of weight there was in particular a significant increase in the amount of 'slow-wave' sleep. Total sleep time greatly 
increased only during the final period of weight gain, and during this period there was a slight decrease in 'slow-wave' sleep and a marked increase in 'rapid-eyemovement (REM)' sleep. On average the latter increased from $99 \mathrm{~min}$ on the first occasion to $109 \mathrm{~min}$ at $15 \%$ below target weight to $139 \mathrm{~min}$ at full target weight. 'REM' sleep still increased by $27 \%$ when a correction was made for increased total sleep time.

We had studied this population at the three body-weights because of our view that, often, important pubertal processes are rekindled at just above the intermediate body-weight (Crisp, 1967a; Palmer, Crisp, Mackinnon, Franklin, Bonnar \& Wheeler, 1975; Crisp, 1977a), at which point there is a sudden shift in the BMR (Crisp, 1970; Crisp \& Kalucy, 1973; Stordy, Marks, Kalucy \& Crisp, 1977). We postulated that we were observing threshold changes in cerebal activity having to do with qualitative changes in growth and energy balance and often mirrored in natural puberty by a surge in bodily growth accompanied by increased carbohydrate intake.

All the anorectic subjects that we studied above had been abstaining anorectics (carbohydrate starved). Next we investigated the impact of various diets on EEG and hormonal sleep (Phillips, Chen, Crisp, Koval, McGuinness, Kalucy, Kalucy \& Lacey, 1975; Chen \& Crisp, 1977) in normal subjects. High carbohydrate-low fat intake was associated with decrease in 'slow-wave' sleep (usually with an accompanying suppression of growth hormone secretion) and increase in 'REM' sleep the next night when compared with the effects of 'balanced' diet and lowcarbohydrate-high-fat isoenergetic diet.

Working experimentally with animals, Jouvet (1967) concluded that 5hydroxytryptamine ( $5-\mathrm{HT})$ might mediate 'slow-wave' sleep and noradrenalin 'REM' sleep. Human experiments, however, have yielded discrepant findings in this area. An increase in 'REM' sleep has in fact been shown to occur after intravenous injection of 5-HT (Mandell, Mandell \& Jacobson, 1965) and oral doses of tryptophan (Hartmann, 1967) in normal adults. Oswald, Berger, Evans \& Thacore (1964) reported that oral administration of tryptophan reduced REM latency. When p-chlorophenylalanine was used to suppress the synthesis of $5-\mathrm{HT}$, 'REM' sleep was reduced (Wyatt, Engelman \& Kupfer, 1969). Nevertheless tryptophan was also found to enhance total sleep time, 'non-REM' sleep and 'deltawave' sleep, suggesting that tryptophan and $5-\mathrm{HT}$ may have different effects on sleep (Wyatt, Engelman \& Kupfer, 1970).

In our next study (Chen, Kalucy, Hartmann, Lacey, Crisp, Bailey, Ecceleston \& Coppen, 1974) we investigated the relationship between free, bound and total plasma tryptophan and the 'sleeping' EEG throughout the night. No direct temporal relationship was found in normal subjects between plasma tryptophan levels and specific sleep stages. What we took to be the nocturnal component of a diurnal variation revealed itself. The mean free plasma tryptophan levels, however, were found to have a positive correlation with 'REM' sleep and a negative correlation with 'non-REM' sleep. These findings were subsequently confirmed in our laboratory in a larger study. 
It must be somewhat speculative at this stage to try to integrate these findings with animal studies, but we have been interested in the work of Wurtman and his laboratory. In 1974 and 1975 (Madras, Cohen, Munro \& Wurtman, 1974; Wurtman \& Fernstrom, 1975) they found that the administration of tryptophan, the injection of insulin or the consumption of a single protein-free, highcarbohydrate meal all increased brain tryptophan levels and soon thereafter the levels of serotonin and its major metabolite 5-hydroxy-indole acetic acid. The addition of protein to the meal suppressed the increase in brain tryptophan and serotonin, probably because protein contributed to plasma considerably larger amounts of the other neutral amino acids (e.g. leucine, phenylalanine) than of tryptophan, these other amino acids competing with tryptophan for uptake into the brain. This group (Wurtman \& Fernstrom, 1974) conclude that diet seems to directly affect cerebral metabolism. They suggest that 'serotonin-releasing brain neurones function as a kind of humoral neural transducer which converts information about peripheral metabolism (as manifested by plasma amino acid pattern) to neural signals (i.e. the release of a greater or lesser quantity of serotonin)'. They hypothesize 'perhaps the brain decides in part when it is hungry or when it is time to sleep, to trigger ovulation or to raise or lower body temperature on the basis of information that it receives about plasma amino acid levels (which in turn depend upon food consumption).... Perhaps nutritional inputs control the (sleeping) brain so that the brain may make appropriate decisions concerning nutritional strategies'. This would accord with a recent finding that the 'sleeping' EEG relates to the next day's dietary intake in cats (Siegel, 1975), with, in general, amounts of 'REM' sleep being negatively correlated with the next day's food intake; and to general theories of sleep (Oswald, 1970) wherein sleep is seen not only as having a restorative function but also as having a re-programming orienting purpose.

Meanwhile, our ongoing studies include the continued investigation of various diets in relation to plasma levels of amino acids and 'EEG' sleep, and also the investigation of food intake, appetite and mood in relation to the pharmacological manipulation of 'EEG' sleep.

In such ways we have been attempting to study aspects of the biochemistry of human sleep. Sleep provides us with a much more experimentally controllable aspect of human growth and energy balance than does wakefulness. Since the two states are, however, interdependent we hope that we shall eventually find ourselves also gaining experimental access to some of the biochemical factors associated with central aspects of wakefulness such as appetite and eating behaviour, perhaps especially through our continued study of people with anorexia nervosa.

\section{REFERENCES}

Anokhin, P. K. (1961). In Brain and Behaviour. [M. A. Brazier, editor]. Washington, DC: American Institute Biological Sciences.

Chen, C. N. \& Crisp, A. H. (1977). Proc. 3rd europ. Sleep Conf., Montpelier, 1976. 
Chen, C. N., Kalucy, R. S., Hartmann, M. H., Lacey, J. H., Crisp, A. H., Bailey, J. E., Eccleston, E. G., \& Coppen, A. (1974). Br. med. F. 4, 564 .

Crisp, A. H. (1967a). f. psychosom. Res. I1, i 17.

Crisp, A. H. (1967b). Hosp. Med. p. 713.

Crisp, A. H. (1970). Wld rez. nutr. Diet. 12, 452.

Crisp, A. H. (1973). Symposium on Anorexia Nervosa and Obesity R. Coll. Physicians, Edinburgh, Publ. no. 42, 18.

Crisp, A. H. (1977a). Int. J. Obesity r, 231.

Crisp, A. H. (1977b). Proc. R. Soc. Med. 70, 464

Crisp, A: H. \& Kalucy, R. S. (1973). Postgrad. med. f. 49, 883.

Crisp, A. H. \& Kalucy, R. S. (1974). Br. f. med. Psychol. 47, 349.

Crisp, A. H. \& Stonehill, E. (1970). F. psychosom. Res. 14, 353.

Crisp, A. H. \& Stonehill, E. (1971). Br. med. f. iii, 149.

Crisp, A. H. \& Stonehill, E. (1973). Br. J. Psychiat. 122, 379.

Crisp, A. H. \& Stonehill, E. (1976). Sleep, Nutrition and Mood. London: Wiley.

Crisp, A. H., Stonehill, E. \& Fenton, G. W. (1970). Psychother. Psychosom. 18, I6r.

Crisp, A. H., Stonehill, E., Fenton, G. W. \& Fenwick, P. C. B. (1973). Psychother. Psychosom. 22, 159.

Hartmann, E. (1967). Psychonomic Sci. 8, 479.

Jouvet, M. (1967). In Sleep and Altered States of Consciousness, chap. 7 [S. S. Kety, E. V. Evarts and H. C. Williams, editors]. Baltimore: Williams and Wilkins.

Kalucy, R. S., Crisp, A. H., Chard, T., McNeilly, A., Chen, C. N., Carruthers, M. \& Zilkha, J. (1977). Lancet i, 447 .

Lacey, J. H., Stanley, P. A., Crutchfield, M. \& Crisp, A. H. (1977). F. psychosom. Res. $21,17$.

MacFadyen, U. M., Oswald, I. \& Lewis, S. A. (1973). F. appl. Physiol. 35, 39 I.

Madras, B. K., Cohen, E. L., Munro, H. N. \& Wurtman, R. J. (1974). Adv. Biochem. Psychopharmac. 11, 143.

Mandell, M. P., Mandell, A. J. \& Jacobson, A. (1965). Rec. Adv. Biol. Psychiat. 7, 115.

Mawson, A. R. (1974). Psychol. Med. 4, 289.

Oswald, I. (1970). New Scientist. 170.

Oswald, I., Berger, R. J., Evans, J. I. \& Thacore, V. R. (1964). EEG clin. Neurophysiol. 17,603.

Palmer, R. L., Crisp, A. H., Mackinnon, P. C. B., Franklin, M., Bonnar, J. \& Wheeler, M. (1975). Br. med. $\mathcal{Y}$. i, 179.

Paykel, E. S., Mueller, P. S. \& de la Vergne, P. M. (1973). Br. F. Psychiat. 123, 501.

Phillips, F., Chen, C. N., Crisp, A. H., Koval, J., McGuinness, B., Kalucy, R. S., Kalucy, E. C. \& Lacey, J. H. (1975). Lancet ii, 723 .

Redmond, D. E. Jr, Huang, Y. H., Baulu, J., Snyder, D. R. \& Maas, J. W. (1977). In Anorexia Nerzosa [R. A. Vigersky, editor]. New York: Raven Press.

Russell, G. F. M. (1970). In Modern Trends in Psychological Medicine, vol. 2 p. 13 I [J. H. Price, editor 7 . London: Butterworths.

Siegel, J. M. (1975). Physiol. Behar. 15, 399.

Slade, P. D. \& Russell, G. F. M. (1973). Psychol. Med. 3, 188.

Stordy, B. J., Marks, V., Kalucy, R. S. \& Crisp, A. H. (1977). Am. J. clin. Nutr. 30, $13^{8}$.

Wurtman, R. J. \& Fernstrom, J. D. (1974). Nutr. Rev. 32, I 93 .

Wurtman, R. J. \& Fernstrom, J. D. (1975). Am. F. clin. Nutr. $28,638$.

Wyatt, R. J., Engelman, K. \& Kupfer, D. J. (1969). EEG clin. Neurophysiol. 27, 529.

Wyatt, R. J., Engelman, K. \& Kupfer, D. J. (1970). Lancet ii, 842.

Young, J. B. \& Landsberg, L. (1977). Science, N.Y. 196, 1473. 